\title{
Provocative testing and drug response in a patient with the long QT syndrome
}

\author{
Mayumi Kawade, Tohru Ohe, Tetsuro Kamiya
}

Department of Cardiovascular Medicine, Okayama University School of Medicine, Okayama, Japan

T Ohe

Department of

Paediatrics, National Cardiovascular Centre, Osaka, Japan T Kamiya

Correspondence to: Dr M Kawade, Department of Paediatrics, Gifu University School of Medicine, 40 Tsukasa machi, Gifu, Japan, 500.

Accepted for publication 2 November 1994

(Br Heart $\mathcal{f} 1995 ; 74: 67-70$ )

Keywords: long QT syndrome, torsades de pointes, isoprenaline.

The idiopathic long QT syndrome (LQTS) is characterised by a prolonged QT interval in the surface electrocardiogram, syncopal episodes, and sudden death caused by ventricular tachyarrhythmia. ${ }^{1-3}$ We describe a patient with LQTS and torsades de pointes. Infusion of isoprenaline or adrenaline induced TU changes and torsades de pointes. We used TU wave changes during isoprenaline infusion to select an effective drug for this patient.

\begin{abstract}
Department of Paediatrics, Gifu University School of Medicine, Gifu, Japan $M$ Kawade

Abstract

A girl of 14 with the long QT syndrome (LQTS) and torsades de pointes is reported. Isoprenaline or adrenaline infusions induced torsades de pointes and inversion of the TU wave. Changes in the TU wave during isoprenaline infusion were used to select effective drugs to treat this patient. A $\beta$ blocker and calcium channel blocker were selected and the patient had no episodes of syncope for two years. This electrocardiographically guided method may be useful for selecting effective drugs in patients with the LQTS.
\end{abstract}

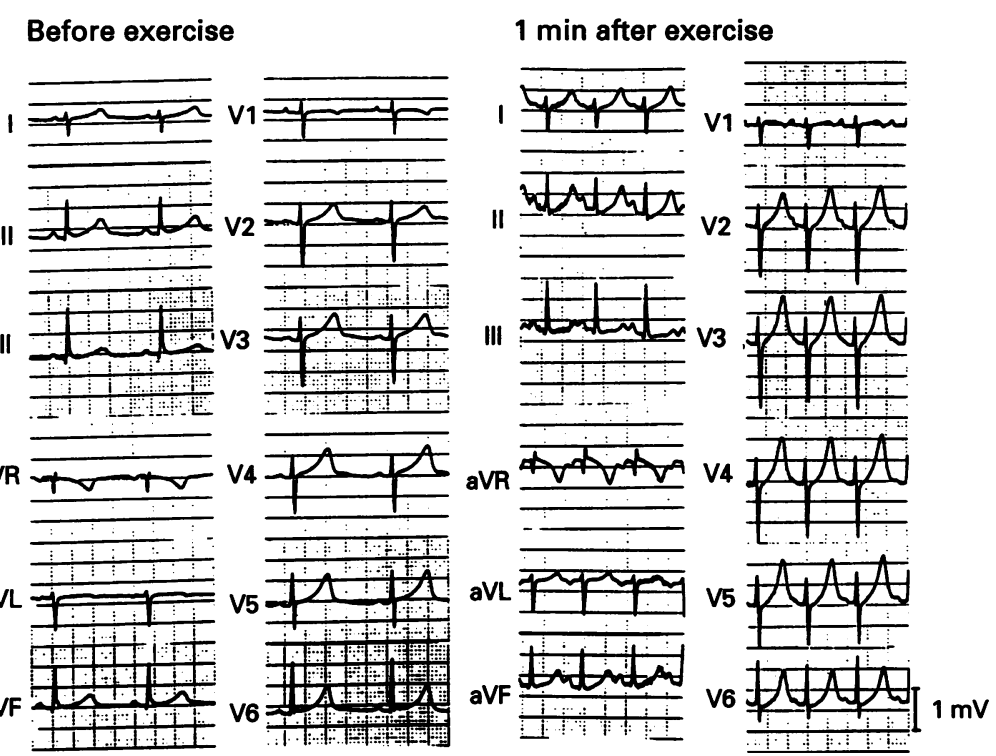

Figure 1 Effects of treadmill testing. One minute after exercise the QTc interval had increased from $460 \mathrm{~ms}$ to $550 \mathrm{~ms}$.
Case report

A 14 year old girl was admitted to the National Cardiovascular Centre because she has had syncopal episodes. She fainted at the age of 13 while being questioned by a teacher at school. She had a second syncopal episode at the same age at school while being scolded by her teacher. She had no neurological abnormality, including deafness. She had never received medical treatment. She had no family history of QT prolongation, syncope, or sudden death.

Physical examination, including neurological examination, on admission showed no abnormal findings. The chest $x$ ray, cross sectional echocardiogram, and serum electrolyte concentrations were normal. The electrocardiogram was obtained on a 6 channel Fukuda Denshi FD-63 at a paper speed of $25 \mathrm{~mm} / \mathrm{s}$ with a calibration of $10 \mathrm{~mm} / \mathrm{mV}$. The QT interval was measured by hand in lead II at rest and during the interventions. The QT interval was corrected for heart rate using Bazzett's formula. The end of the $T$ wave was defined as the return to the baseline. When the first component of the $T$ wave and the second component of the $T$ wave ( $U$ wave) fused, making it impossible to distinguish two types of waves, the QT interval was measured to the time of final return to the baseline. The QT interval of the resting ECG was $450 \mathrm{~ms}$ at a heart rate of 64 beats $/ \mathrm{min}(\mathrm{QTc}=460 \mathrm{~ms}$ ).

\section{EXERCISE TESTING}

Treadmill testing was performed according to a modified Bruce protocol (fig 1). The protocol stopped when the patient became exhausted. ECG was obtained on a six channel Marquette CASE 12 at a paper speed of 25 $\mathrm{mm} / \mathrm{s}$ with a calibration of $10 \mathrm{~mm} / \mathrm{mV}$. The QT intervals were measured in lead II. Heart rate increased from 70 to 143 beats $/ \mathrm{min}$ with exercise. QTc was prolonged from $460 \mathrm{~ms}$ to $550 \mathrm{~ms}$

RESPONSES TO ADRENALINE, ISOPRENALINE AND METHOXAMINE INFUSION

To examine the influence of alpha and/or beta adrenoreceptor stimulation, adrenaline, isoprenaline, and methoxamine were administered by infusion after we had obtained informed consent from her parents (fig 2). During adrenaline infusion at $0.4 \mu \mathrm{g} / \mathrm{kg} / \mathrm{min}$ (alpha and beta stimulation) the QTc interval was prolonged (from $470 \mathrm{~ms}$ to $600 \mathrm{~ms}$ ) and prominent $U$ waves developed especially in lead V3. A bolus injection of adrenaline $(0 \cdot 8$ $\mu \mathrm{g} / \mathrm{kg}$ ) induced inversion of the TU wave in the left precordial leads, multiform premature 
Figure 2 Responses to adrenaline, isoprenaline, and methoxamine infusion. Administration of adrenaline or isoprenaline was associated with the prolongation of the $Q T$ interval, the development of prominent $U$ waves, and slow VT. Methoxamine increased $U$ wave amplitude. The changes were less pronounced than those produced by adrenaline or isoprenaline.

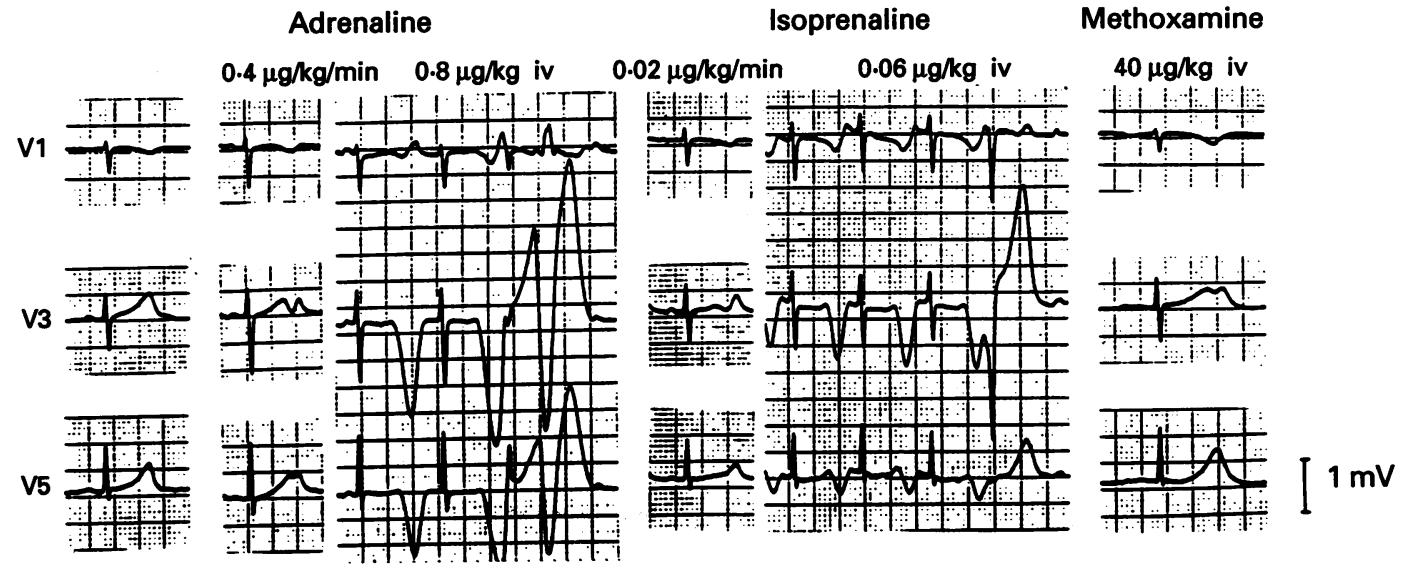

Adrenaline

\}

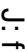

$\stackrel{\overrightarrow{\bar{s}}}{\underline{\mathrm{o}}}$

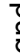

ventricular contractions (PVCs), and slow ventricular tachycardia. Infusion of adrenaline (beta stimulation) at the rate of 0.02 $\mu \mathrm{g} / \mathrm{kg} / \mathrm{min}$, prolonged the QTc interval (from $480 \mathrm{~ms}$ to $590 \mathrm{~ms}$ ) and induced large $U$ waves. A bolus injection of adrenaline $(0.06$ $\mu \mathrm{g} / \mathrm{kg}$ ) was associated with inversion of the TU wave in the left precordial leads and multiform PVCs. After bolus injection of methoxamine $(40 \mu \mathrm{g} / \mathrm{kg}$, alpha stimulation) there were increases in blood pressure (from 105/60 $\mathrm{mm} \mathrm{Hg}$ to $128 / 82 \mathrm{~mm} \mathrm{Hg}$ ), in QTc interval (from $470 \mathrm{~ms}$ to $560 \mathrm{~ms}$ ), and in $U$ wave amplitude.

ELECTROPHYSIOLOGICAL STUDY

After informed consent was obtained from her parents, we performed an electrophysiological study with the patient fasting, and not (MAPs) were recorded as previously reported. ${ }^{45}$ MAPs were recorded from the right ventricular septum (RVS) and the right ventricular anterior wall (RVA). The duration of MAPs was measured at $90 \%$ repolarisation $\left(\mathrm{MAPD}_{90}\right)$. Adrenaline was infused at the same protocol as described. After drip infusion, the $U$ wave became larger than the $T$ wave and the QT interval was prolonged. MAPD $_{90}$ increased from $280 \mathrm{~ms}$ to $420 \mathrm{~ms}$ at the RVS and from $290 \mathrm{~ms}$ to $350 \mathrm{~ms}$ at the RVA. During drip infusion of adrenaline the patient became uncomfortable. Rapid injection of adrenaline induced torsades de pointes. Humps, which were probably consistent with early afterdepolarisations, were seen immediately before the onset of torsades de pointes (fig 3 ). sedated. Monophasic action potentials
Figure 3 Recording of monophasic action potential after drip infusion and additional injection of adrenaline. The bolus injection of adrenaline was associated with torsades de pointes. The inversion of $T U$ wave, the $O T$ prolongation, and humps appeared immediately before the onset of torsades de pointes. $M A P$, monophasic action potential.
Adrenaline $\quad 0.4 \mu \mathrm{g} / \mathrm{kg} / \mathrm{min} \longrightarrow 0.8 \mu \mathrm{g} / \mathrm{kg}$ iv

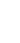

1

II

III

V1

V3

V5

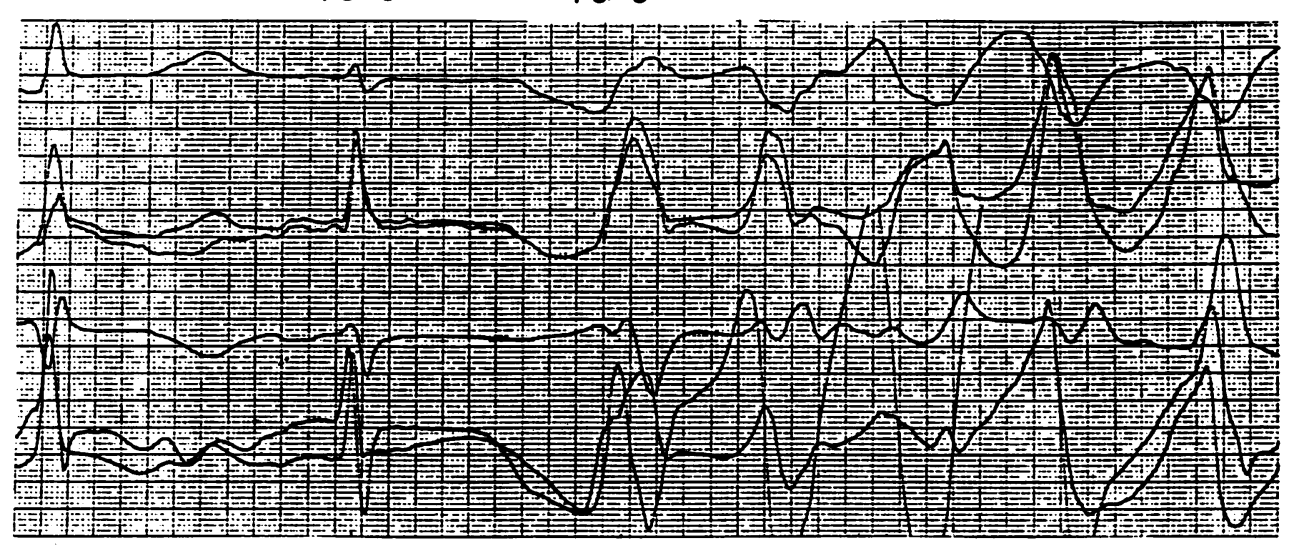

RVS
MAP

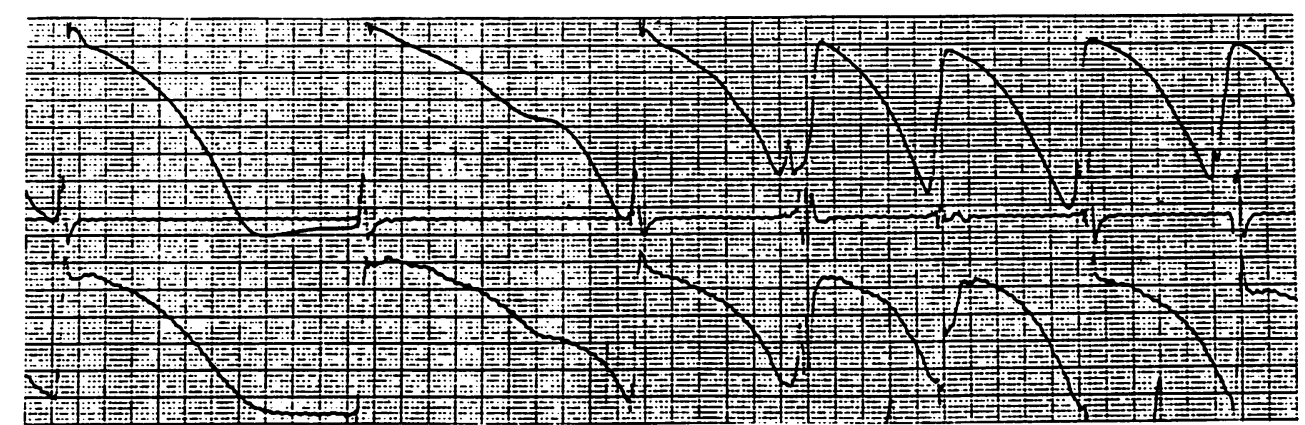




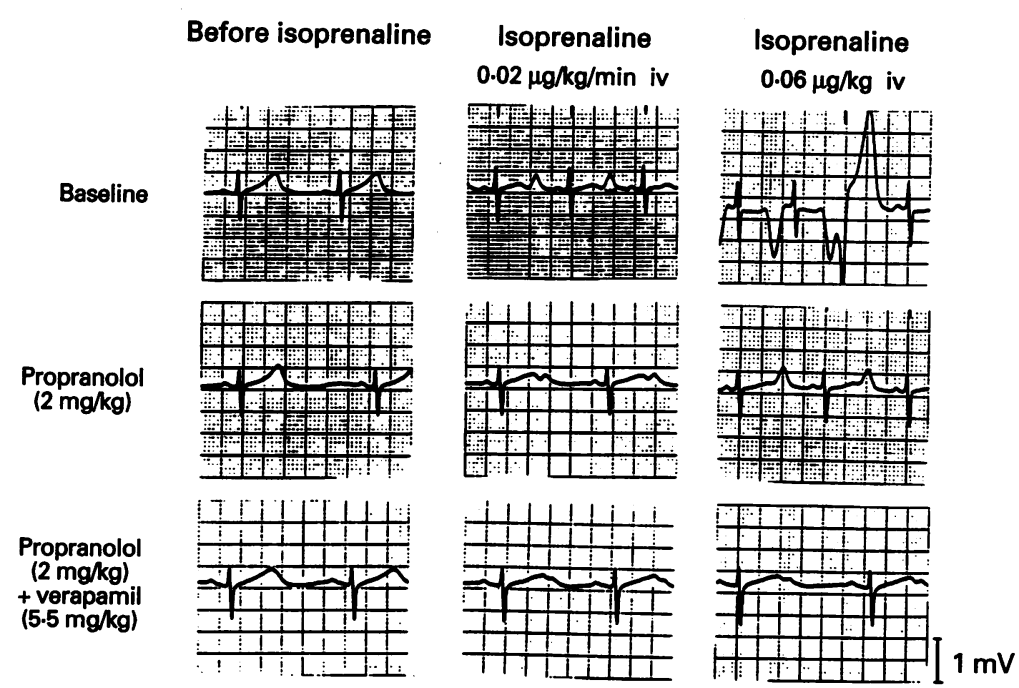

Figure 4 Effects of propranolol and verapamil on preventing the changes in TU produced by isoprenaline. Without such medication $Q T c$ was prolonged and $U$ wave amplitude was much increased during isoprenaline infusion. An additional bolus injection of isoprenaline resulted in the inversion of TU waves and premature ventricular contractions. Propranolol prevented the $T U$ alternans waves and suppressed the increase in $U$ wave amplitude during drip infusion of isoprenaline. After bolus injection of isoprenaline premature ventricular contractions were prevented but prominent $U$ waves were observed. Propranolol with verapamil suppressed the TU changes and ventricular extrasystoles more effectively than propranolol alone. was difficult to estimate the effects of these drugs by the change in QTc interval or the configuration of TU waves.

This patient continued to receive propranolol and verapamil and has not had a syncope attack for 2 years.

\section{Discussion}

In this patient prominent $U$ waves, inversion of the TU wave, PVCs, and non-sustained VT were reproducibly induced by administration of adrenaline or isoprenaline. Beta blockers are effective in $75 \%-80 \%$ of symptomatic patients with idiopathic long QT syndrome. ${ }^{6-8}$ Previously, the effect of drugs has been estimated by their clinical effects. However, in this patient, it was difficult to estimate the drug effect before discharge. Therefore, we used the TU changes to select an effective drug. During drip infusion of isoprenaline, propranolol suppressed the TU changes, but propranolol and verapamil prevented the augmentation of the $U$ wave more effectively.

Figure 3 shows early afterdepolarisations immediately before the onset of torsades de pointes. This suggests a correlation between these two features. When each afterdepolarisations appeared the TU wave became significantly inverted. Morganroth suggested that $U$ waves may represent a summation of early afterdepolarisations occurring throughout the myocardium. ${ }^{9}$ Moore reported that early afterdepolarisations may stem from increased inward calcium or sodium currents or from decreased outward potassium current. Early afterdepolarisations may occur when inward depolarising currents exceed outward repolarising currents. In contrast to the normal recovery process, early afterdepolarisations are associated with additional action potentials occurring during the repolarisation process; the rapidly occurring early afterdepolarisations may trigger malignant arrhythmias. These findings suggest that by blocking the calcium current during the repolarisation process, verapamil can suppress the formation of early afterdepolarisations and so abolish TU changes.

A limitation of this report is that we could not document spontaneous torsades de pointes in this patient. We had evidence of only two episodes of syncope, both after emotional stress. We have no evidence that the syncope was caused by torsades de pointes. However, we could induce multiple premature ventricular contractions and TU changes with isoprenaline or adrenaline infusions. Furthermore, propranolol and verapamil prevented multiple premature ventricular contractions and the TU changes induced by isoprenaline or adrenaline infusion.

After starting treatment with propranolol and verapamil she has been free of attack for two years. The electrocardiographically guided selection of the effective drugs may be useful for patients with idiopathic long QT syndrome. 
1 Jervell A, Lange-Nielse F. Congenital deaf-mutism, functional heart disease with prolongation of the QT interval and sudden death. Am Heart 7 1957;54:59-68.

2 Schwartz PJ. Idiopathic long QT syndrome: Progress and questions. Am Heart 7 1985;109:399-411.

3 Gillette PC, Garson A. Ventricular arrhythmias. Pediatric arrhythmias: electrophysiology and pacing. Philadelphia: WB Saunders, 1990:468-72.

4 Ohe T, Kurita T, Aihara N, et al. Electrocardiographic and electrophysiologic studies in patients with torsades de pointe. Role of monophasic action potentials. $\mathcal{F p}_{\mathrm{p}}$ Circ $\mathcal{F}$ 1990;54:1323-30.

5 Shimizu W, Ohe T, Kurita T, et al. Early afterdepolarizations induced by isoproterenol in patients with congenital long QT syndrome. Circulation 1991;84:1915-23.
6 Jackman WM, Friday KJ, Anderson JL. The long QT syndromes: a critical review, new clinical observations and a unifying hypothesis. Prog Cardiovasc Dis 1988;31: 115-72.

7 Schwartz PJ, Locati E. The idiopathic long QT syndrome. Pathogenetic mechanisms and therapy. Eur Heart $\mathfrak{f}$ 1985;6(suppl.D):103-14.

8 Emanuela HL, Schwartz PJ. The idiopathic long QT syndrome: therapeutic management. PACE 1992;15 1374-9.

9 Morganroth J. Relations of QTc prolongation on the electrocardiogram to torsades de pointes: Definitions and mechanisms. Am f Cardiol 1993;72:10B-13B.

10 Moore EN. Mechanisms and models to predict a QT effect. Am $\mathcal{f}$ Cardiol 1993;72:4B-9B.

\section{IMAGES IN CARDIOLOGY}

\section{"Sago" in a saphenous vein graft}

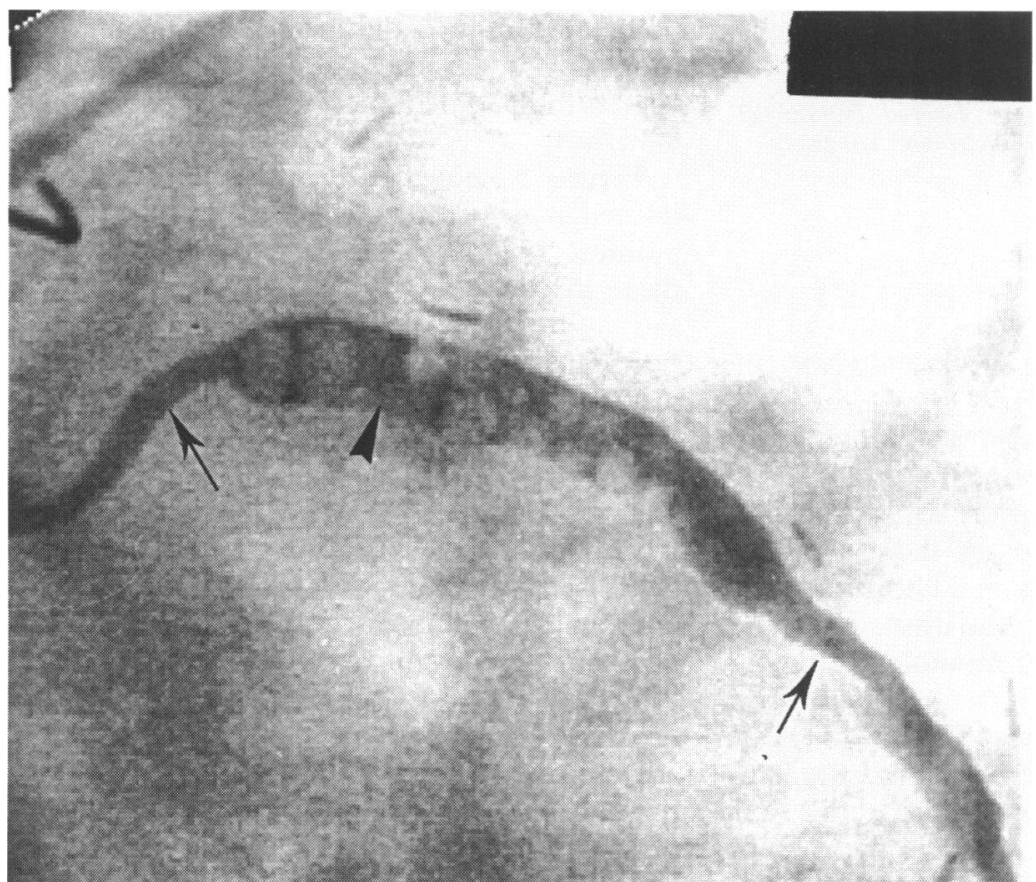

A 65 year old man was free of angina after coronary artery bypass grafting. Angina recurred 57 months later and rapidly worsened over the next 3 months. Coronary and graft angiography was performed and selective angiography of a reversed saphenous vein graft to the obtuse marginal branch of the circumflex showed severe stenoses in the proximal and middle segments of the graft (arrows). The segment between the stenoses contained multiple, mobile, oscillating filling defects suggestive of thrombus (large arrowheads). There are clips on the left internal mammary artery graft (small arrowhead).

JANE S SKINNER PHILIP C ADAMS

Figure 1 Left anterior oblique view of the proximal part of the circumflex graft (digitally magnified $\times 2$ ).

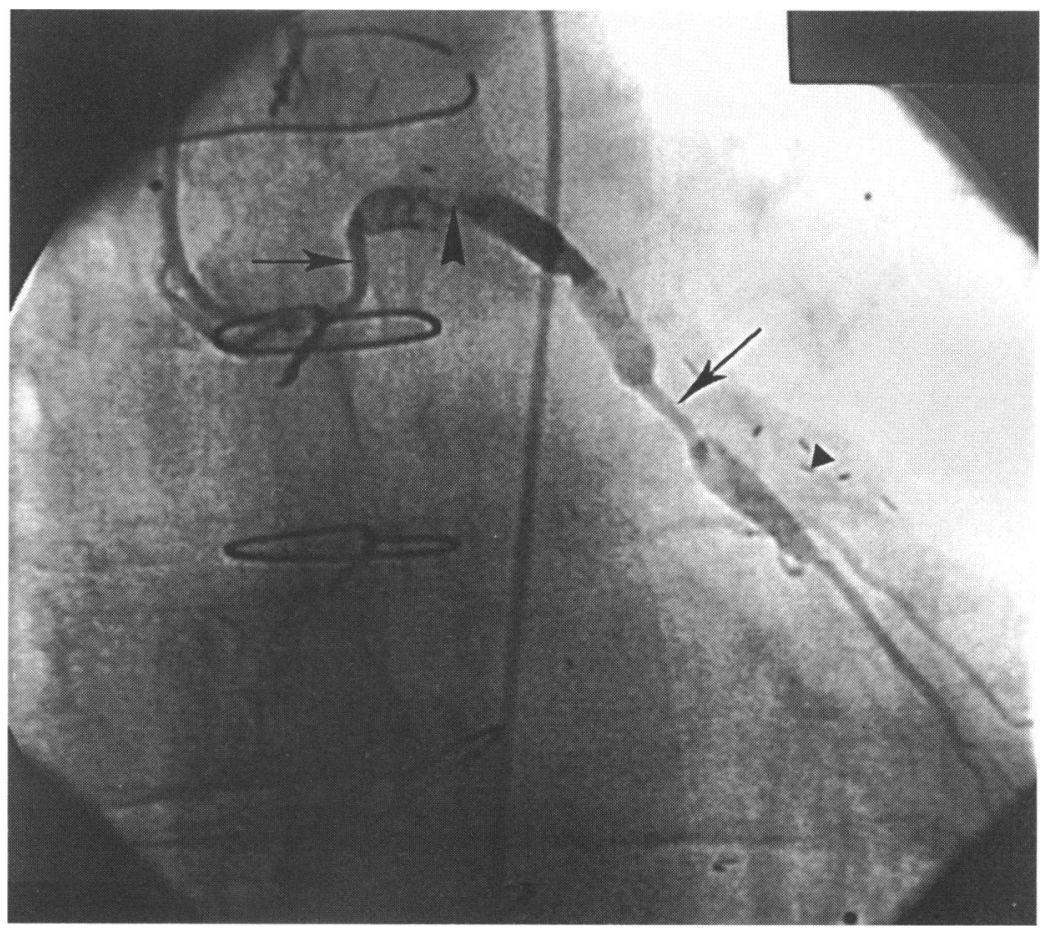

